\title{
Metastatic Ovarian Cancer
}

\author{
DEEPAK DABKARA \& ATUL SHARMA
}

Secondary ovarian cancer results from metastasis from a primary cancer elsewhere in the body \& accounts for about $5 \%$ of ovarian cancer . A Krukenberg tumour classically refers to a secondary ovarian malignancy whose primary site arose in the gastrointestinal tract. Stomach is the primary site in most Krukenberg tumour cases $(70 \%)$. Carcinomas of colon, appendix, and breast (mainly invasive lobular carcinoma) are the next most common primary sites. Rare cases of Krukenberg tumour originating from carcinomas of the gallbladder, biliary tract, pancreas, small intestine, ampulla of Vater, cervix, and urinary bladder/urachus have been reported.

The interval between the diagnosis of a primary carcinoma and the subsequent discovery of ovarian involvement is usually 6 months or less, but longer periods have been reported. In many cases, the primary tumour is very small and can escape detection. A history of a prior carcinoma of the stomach or another organ can be obtained in only $20 \%$ to $30 \%$ of the cases.

Women with Krukenberg tumours tend to be young, with an average age of 45 years.

Patients with krukenberg tumour can be asymptomatic or present with abdominal pain and distension, or with non-specific gastrointestinal symptoms. In addition, Krukenberg tumour is reportedly associated with virilization resulting from hormone production by ovarian stroma. Ascites is present in $50 \%$ of the cases and usually reveals malignant cells.

Radiologically, Krukenberg tumours on USG \& CT scans usually appear as bilateral ovarian masses in more than $80 \%$ cases. The masses are usually solid but can also be cystic. The ovaries are usually asymmetrically enlarged, with a

Department of Medical Oncology

All India Instutute of Medical Sciences, New Delhi-29

E-mail : atul1@hotmail.com bosselated contour.

\section{DIFFERENTIAL DIAGNOSIS}

The diagnosis of Krukenberg tumours largely depends on the recognition of its characteristic light microscopic features with hematoxylineosin-stained sections. However, Krukenberg tumours may mimic other metastatic or primary ovarian tumours. Distinction from the latter is of great importance as misclassification of Krukenberg tumour as a primary ovarian tumour may lead to suboptimal treatment of the patient.

IMMUNOHISTOCHEMISTRY IN THE DISTINCTION OF METASTATIC CARCINOMAS FROM PRIMARY OVARIAN NEOPLASMS

Immunohistochemical evaluation may aid in distinguishing primary ovarian carcinomas from metastatic carcinomas. Cytokeratins 7 and 20 (CK7 and CK20) immunophenotype is the most commonly used analysis. Primary ovarian carcinomas are almost always immunoreactive to CK7 $(90 \%-100 \%)$ but generally are not immunoreactive to CK20. By contrast, metastatic gastric carcinoma tends to be less frequently positive for CK7 (55\%) but is positive for CK20 in approximately $70 \%$ of cases. Colorectal adenocarcinomas are usually negative for CK7 but positive for CK20 in most cases. Tumours metastasizing from the appendix are commonly positive for CK20 but positive also for CK 7 in $50 \%$ of cases. Therefore, a $\mathrm{CK} 7^{+} / \mathrm{CK} 20$ immunophenotype favors a primary ovarian carcinoma, whereas a CK7 $/ \mathrm{CK} 20^{+}$or $\mathrm{CK} 7^{+} / \mathrm{CK} 20^{+}$ immunophenotype (CK20 positivity, in particular) favors a metastatic gastrointestinal carcinoma.

Use of source-specific antibodies can increase the diagnostic confidence. For example, immunoreactivity for carcinoembryonic antigen and CDX2 together with the immunoexpression pattern of $\mathrm{CK} 7 \% \mathrm{CK} 20^{+}$increases the confidence in pointing toward the colorectal origin of the pri- 
mary.

PROGNOSIS AND MANAGEMENT

Patients with Krukenberg tumours have an overall mortality rate that is significantly high. Most patients die within 2 years (median survival, 14 months).Review of the literature shows that the prognostic factors for Krukenberg tumours have not been well established, Several studies show that the prognosis is poor when the primary tumour is identified after the metastasis to the ovary is discovered, and the prognosis becomes worse if the primary tumour remains covert. No optimal treatment strategy for Krukenberg tumours has been clearly established in the literature. Since the Krukenberg tumour is a secondary (metastatic) tumour, management of the tumour must involve finding and treating the primary cancer. In general, most cases of Krukenberg tumour have a poor prognosis and radical operation such as removal of the ovaries (and the colon or appendix if involved) can improve survival only in cases of solitary ovarian metastasis or local extended disease (i.e. the lesion is located only in the pelvis). Cancer chemotherapy and radiotherapy before surgery may be used to shrink the tumour and facilitate its removal.

\section{CONCLUSIONS}

Krukenberg tumour is a metastatic ovarian tumour that is histologically characterized by mucin-laden signet ring cells. Stomach is the most common primary site, but other organs can serve as a primary site. The lymphatic system is the most likely route for metastasis. Diagnosis of Krukenberg tumour with unknown primary warrants careful investigation of mainly the digestive tract and other potential sites. CA 125 levels can be used for screening for early detection of ovarian metastasis as well as for monitoring the course of disease. The prognosis of Krukenberg tumour is poor and no curative treatment is currently available. 